Systemy Logistyczne Wojsk nr 51/2019

\title{
MODEL OF FUNCTIONING OF INFORMATION-CONTROL SYSTEM OF UNMANNED CAR WITH DIFFERENT SENSORS CHANNELS OF INFORMATION INTERACTION
}

\author{
Viktor KLIMENKO \\ https://orcid.org/0000-0002-8073-4404 \\ Oleg SUKHIN \\ Denis KOTOV \\ https://orcid.org/0000-0002-6775-5593 \\ Denis LISOVENKO \\ Odesa Military Academy \\ Odesa, Ukraine
}

\begin{abstract}
The article proposes an approach to the formalization of the process of information interaction of the information and control system of an unmanned vehicle with the environment, taking into account external destabilizing effects against the background of intra-system interference. The result of simulation modeling of the process of inverse processing of information arrays under conditions of a priori uncertainty is presented.
\end{abstract}

Keywords: information system, unmanned vehicle, factors of destabilization, a priori uncertainty, interference, analytical model, simulation

\section{INTRODUCTION}

The problem of the qualitative functioning of a robotic vehicle, which is operated within the framework of "car - environment of operation with objects" is characterized by uncertainty and conflict of conditions of its operation.

The uncertainty and conflict of the environment of operation largely depend on the intensity of the information interaction between the car, the object of robotics, and objects of the environment of its operation, as well as directly from the properties of the information itself.

Modern approaches to assessing the quality of information streams processed in the information-control system (ICS) with unmanned cars are based on the methods of processing digital (digitized) information. It is digitization, as the process of processing the input information, as well as its multi-sensory nature, make mistakes (inaccuracy of measurement) of the entire input information array, which in turn leads to a decrease in the efficiency of the operation of the information-controlled control system of an unmanned car in uncertain and conflict conditions of its exploitation. 
Therefore, the formalization of the information interaction processes of the ICSU of a robotic vehicle in unorganized environments and non-deterministic operating situations remains an urgent task.

The purpose and formulation of the research task. The purpose of the work is to evaluate the effectiveness of the information and control system (ICS) functioning of the ground robotic complex management in unorganized environments and non-deterministic situations. The task is a formal description of the interaction process of objects, criteria for evaluating the effectiveness, and rules for the optimal or adaptive operation of operations provided that an unmanned vehicle is operated in an organized and conflict environment.

\section{MODELING OF PROCESSES OF FUNCTIONING OF A ROBOTIC (SELF- CONTROLLED) CAR WITH MULTI-SENSORY INFORMATION COMMUNICATION CHANNELS}

In the context of setting the task for the study of systemic importance issues of the domain formalization. The formalization system involves the definition of a process model of observation and the use of language spatially-temporal description of the conditions of the operation $w \in W$, condition of the robotics $\mathbf{\Xi}$, and the influence on it of external and internal disturbances.

Accepted in the process model approach comes from the chosen efficiency criterion $J_{G}$ or a given target function in the next information-probabilistic interpretation of the output data.

In particular:

- a set of a priori unknown parameters of the process of technological interaction of a multi-sensory system of the ICS of RC with electromagnetic sources of information of the environment of operation is represented by the $\mathrm{m}$-dimensional $\mathbf{X}=\mathbf{X}_{\mathrm{m} \times 1}$. The connection to the real situation assumes the existence of external obstacles $\boldsymbol{\eta}(\mathbf{t})$ and a carrier of information parameters, the socalled useful signal $\mathbf{S}(\mathrm{t})$, which are described by k-dimensional vector $\boldsymbol{\eta}=\boldsymbol{\eta}_{\mathbf{k} \times \mathbf{1}}$; with $\mathbf{m}_{\boldsymbol{\eta}}=$ $\mathbf{M}(\boldsymbol{\eta})=\mathbf{0}$ i $\mathbf{A}_{\boldsymbol{\eta}}=\mathbf{M}\left(\boldsymbol{\eta} \boldsymbol{\eta}^{\mathbf{T}}\right)$ and k-dimensional vector $\mathbf{S}=\mathbf{S}_{\mathbf{k} \times \mathbf{1}}$ 3 $\mathbf{m}_{\mathbf{S}}=\mathbf{M}(\boldsymbol{S})$ i $\mathbf{A}_{\mathbf{s}}=\mathbf{M}\left(\mathbf{S S}^{\mathbf{T}}\right)$, in accordance;

- the result of observation of the interaction process of the multisensory system of independent sensors $\boldsymbol{D}_{\boldsymbol{x}}$ object robotizing $\boldsymbol{\Xi}$ with a complex of incoming implementations $\boldsymbol{X}$ is described by the rules of vector-matrix transformations:

$$
\mathrm{U}=\mathrm{D}_{\mathrm{x}}(\mathrm{S}+\eta)+\mathrm{n}
$$

where:

- $\quad \boldsymbol{D}_{\boldsymbol{x}}=\boldsymbol{D}_{\boldsymbol{x}(m \times k)}$ - matrix of transfer functions of multi-sensory sensor systems, 
- $\boldsymbol{n}=\boldsymbol{n}_{m \times 1}-m$-dimensional vector of the internal noise sensor system (independent measurement errors), which has zero expectation $\mathbf{m}_{\mathbf{n}}=\mathbf{M}(\mathbf{n})=\mathbf{0}$ and unit matrix of correlation $\mathbf{A}_{\mathbf{n}}=\mathbf{M}\left(\mathbf{n n}^{\mathbf{T}}\right)=\mathbf{I}$.

- it is assumed that the vectors of the useful signal $\boldsymbol{S}$, external interference $\boldsymbol{\eta}$ and the internal noise

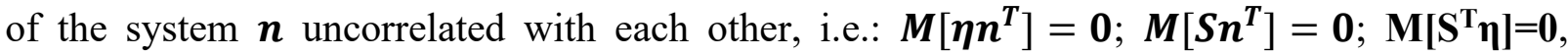
де $\mathbf{0}=\mathbf{0}_{k \times m}-$ rectangular zero $(k \times m)$ - dimensional matrix.

The indicated information-probabilistic interpretation of the data model takes into account the redundancy of the model version of RC and the excessive environment of its exploitation. Information redundancy is introduced as a result of observation $\boldsymbol{U}$ so, to evaluate the parameters $\hat{S}$ unknown vector $\boldsymbol{S}$ satisfies the criterion of optimality in the Euclidean metric, in particular, the minimum of the meansquare error of approximation:

$$
\boldsymbol{E}=\boldsymbol{M}\{\|\boldsymbol{S}-\hat{\mathrm{S}}\|\}=\min
$$

where: $\|\bullet\|-$ the norm of a vector describing the parameters of a useful signal.

Estimation of parameters $\hat{S}$ is in the class of linear transformations by combining (complexing) the results of observation $\boldsymbol{U}$ at the output of the system of independent multisensor sensors according to the following algorithm:

$$
\hat{S}=F_{D}^{-1}(U)=F_{D}^{-1}[F(x)]=W_{x} U
$$

where:

○ $\boldsymbol{F}_{\boldsymbol{D}}{ }^{-1}(\bullet)$ - operator transformation vector observations $\mathbf{U}$,

○ $\boldsymbol{W}_{\boldsymbol{x}}=\boldsymbol{W}_{\boldsymbol{x}(\boldsymbol{k} \times \boldsymbol{m})}-$ matrix transfer characteristic of the system of combining the results of observation $\mathbf{U}$, which is optimal for the criterion (2).

The schematic interpretation of the algorithms (1) and (3) in the generalized form is shown in Fig. 1.

The generalization of this scheme is that in it, along with external factors $\boldsymbol{\eta}$, are reflected and intrasystem factors of destabilization $\boldsymbol{\Delta W}$, which in algorithms (1) and (3) are currently absent.

Definition of transmission characteristics of the system $\boldsymbol{W}_{\boldsymbol{x}}$ is based on the orthogonality condition of the result vector of the observation $\boldsymbol{U}$ and the vector of independent measurement errors $\boldsymbol{E}=\boldsymbol{S}-\hat{\mathrm{S}}:$

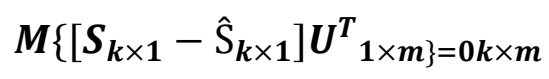

Substituting to (3) the value of the estimate (4), we obtain an equation system for determining the optimal transmission characteristic $\boldsymbol{W}_{\boldsymbol{x}}$ :

$$
\boldsymbol{M}\left\{[\boldsymbol{S}-\hat{\mathrm{S}}] \boldsymbol{U}^{T}=\boldsymbol{W}_{x} \boldsymbol{M}\left\{\boldsymbol{U} \boldsymbol{U}^{T}\right\}\right.
$$


The solution of this system of equations is a classical inverse problem, the results of which determine the transmission characteristic of the system of the association of multi-sensory information channels, in particular:

$$
W_{x(k \times m)}=a_{k \times m} A^{-1}{ }_{m \times m}
$$

where:

○ $\boldsymbol{A}_{\boldsymbol{m} \times \boldsymbol{m}}=\boldsymbol{M}\left\{\boldsymbol{U}_{\boldsymbol{m} \times \mathbf{1}} \boldsymbol{U}^{\boldsymbol{T}}{ }_{\mathbf{1} \times \boldsymbol{m}}\right\}$ - correlation matrix of the observation process,

○ $\boldsymbol{a}_{\boldsymbol{k} \times \boldsymbol{m}}$ - rectangular matrix of reference vectors in the form:

$$
a_{k \times m}=\left[\begin{array}{ll}
a_{1}^{T} & (1 \times m) \\
a_{2}^{T} & (1 \times m) \\
\vdots & \\
a_{k_{1}}^{T} & (1 \times m)
\end{array}\right]
$$

Substituting the expression (5) to (3), we obtain the value of the linear unbiased estimation of the vector of the useful signal:

$$
\hat{S}=\hat{S}_{k \times 1}=a_{k \times m} A^{-1}{ }_{m \times m} U_{m \times 1}
$$

with a minimum measurement error $\mathbf{E}_{\mathbf{m i n}}=\mathbf{S}-\hat{\mathrm{S}}=\mathbf{S}-\mathbf{a} \mathbf{A}^{-\mathbf{1}} \mathbf{U}$.

Correlation matrix of measurement error $\boldsymbol{E}$ taking into account the previously determined statistical moments has the following form:

$$
M\left(E E^{T}\right)=A_{S}-a A^{-1} a^{T}
$$

There is a correlation matrix $\operatorname{tr}\left\{\boldsymbol{M}\left(\boldsymbol{E} \boldsymbol{E}^{\boldsymbol{T}}\right)\right\}$ expresses the least possible of the mean square error: $\boldsymbol{\varepsilon}=$ $\operatorname{tr}\left\{M\left(E E^{T}\right)\right\}$.

The model variant of the combination according to the algorithm (8) of the multi-sensory channels of the information system of the ICS of RC in conditions of a priori uncertainty of its environment, is presented in Fig. 3 .

Reflecting the optimal data structure, the model variant of the merger algorithm (Figure 3) involves the operation of "bleaching" $\boldsymbol{A}^{-\mathbf{1}}{ }_{\boldsymbol{m} \times \boldsymbol{m}}$ noisy implementations at the output of the sensor system $\boldsymbol{U}_{\boldsymbol{m} \times \mathbf{1}}$ and the operation of comparing the result with the vector of the standards $\boldsymbol{a}_{\boldsymbol{m} \times \boldsymbol{k}}$ (prior images). As a result of these operations, an optimal estimate is formed $\hat{S}_{\boldsymbol{k} \times \mathbf{1}}$ vector state of the environment. Optimum vector estimation $\hat{S}_{\boldsymbol{k} \times \mathbf{1}}$ analyze in accordance with three situations:

- the first situation is the number of measurements $m$ (sensors of information) is less than the number of measuring parameters $k$, that is $m<k$;

- $\quad$ second situation - the number of measurements $m$ (sensors of information) more than the number of measuring parameters $k$, that is $m>k$;

- the third situation - the number of measurements $m$ (sensors of information) and is equal to the number of measuring parameters $k$, that is $m=k$. 
The research of these situations is carried out based on the relative estimation of the mean square error of the vector parameters $\boldsymbol{S}_{\boldsymbol{k} \times \boldsymbol{x}}$ :

$$
\breve{\varepsilon} \frac{\operatorname{tr}\left\{M\left(E E^{T}\right)\right\}}{\|S\|^{2}} \frac{M\left\{\|S-\breve{S}\|^{2}\right\}}{\|S\|^{2}} \text { min }
$$

For the first situation, when the redundancy of the sensor system is absent $m<k$, estimation mean square error is given by:

$$
\breve{\varepsilon}(m<k) \frac{1}{q_{s}}\left[D x_{(m \times k)} D_{X}^{T}(k \times m)\right]_{\text {min }}^{-1}
$$

where: $q_{s}-$ signal/noise ratio in the information channel.

In an alternative situation, when there is a redundancy of sensor systems $m>k$, the estimation of the mean-square error becomes of such significance:

$$
\breve{\varepsilon}(m>k) \frac{1}{q_{s}}\left[D_{X}^{T}{ }_{(k \times m)} D x_{(m \times k)}\right]_{\text {min }}^{-1}
$$

For the situation, $m=k$ estimation mean square error is:

$$
\breve{\varepsilon}(m=k) \frac{1}{q_{s}}\left[D_{X_{(m \times m)}}^{-1} D_{X_{(m \times m)}}^{-1}\right]_{\min }=\frac{1}{q_{s}}\left\|D_{X_{(m \times m)}}^{-1}\right\|^{2}
$$

After completing several standard transformations and transformations from (11) - (13), we obtain a system of equations for situational analysis of the estimation of the mean square error in the conditions of the influence of destabilizing factors of external and intersystem origin.

In particular:

$$
\begin{gathered}
\left.\breve{\varepsilon}_{\min }\right|_{(m>k)}=(k-m)+\frac{q_{\eta}}{q_{s}} \operatorname{tr}\left[D_{X_{(m \times k)}} D_{X_{(k \times m)}}^{T}\right]^{-1}+\left.\sigma_{W_{x}}^{2} f(m, k)\right|_{(m<k)} \\
\left.\breve{\varepsilon}_{\min }\right|_{(m>k)}=\frac{q_{\eta}}{q_{s}} \operatorname{tr}\left[D_{X_{(k \times m)}^{T}}^{T} D_{X_{(m \times k)}}\right]^{-1}+\left.\sigma_{W_{x}}^{2} f(m, k)\right|_{(m>k)} \\
\left.\breve{\varepsilon}_{\min }\right|_{(m=k)}=\frac{q_{\eta}}{q_{s}}\left\|D_{X_{(m \times m)}}^{-1}\right\|^{2}+\left.\sigma_{W_{x}}^{2} f(m, k)\right|_{(m=k)}
\end{gathered}
$$

where:

- $q_{\eta}$ - excess of the power of the interference of the environment above the level of power of the internal noise of the sensor system,

- $\sigma_{\mathrm{W}_{\mathrm{x}}}^{2}$ - the relative level of destabilizing intra-system perturbations,

- $f(m, k)$ - the function of the sensitivity of the system of information sensors to destabilizing intra-system disturbances.

The results of simulation (Fig. 1-4) indicate the adequacy of the proposed approach to the formalization of the information interaction processes of the ICS with the environment of exploitation 
with objects in the situation of redundancy of the information system (random perturbations) under conditions of multilevel influence of external destabilizing factors of artificial and natural, as well as destabilizing factors of intra-system origin, objectively present in any information system.

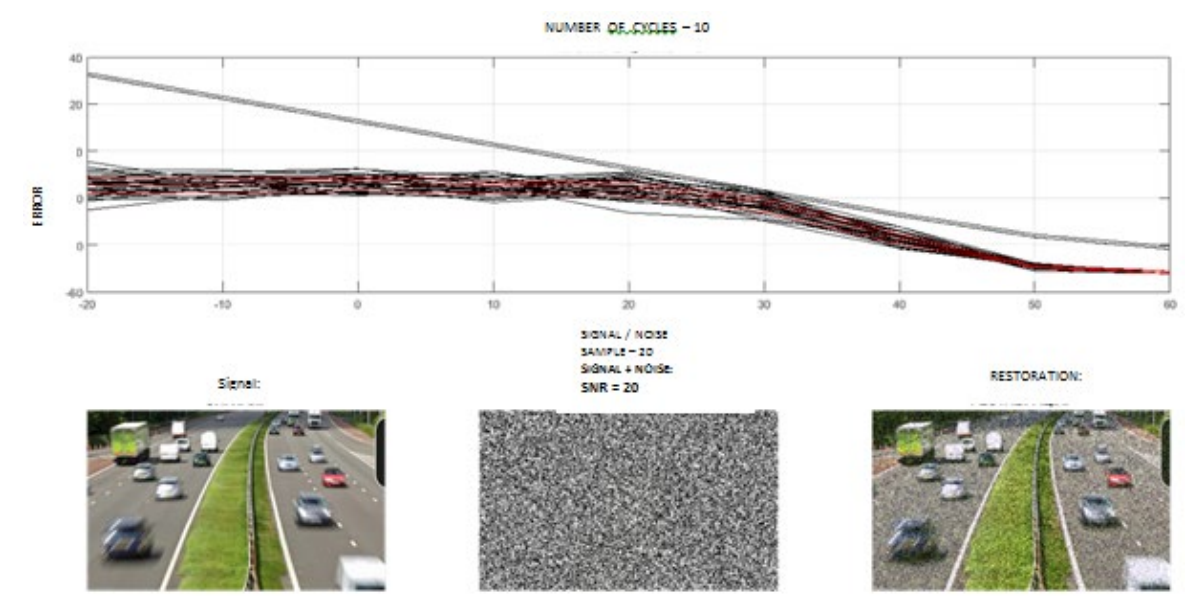

Fig. 1. Results of simulation of the functioning of the ICS RC in MATLAB environment Source: Drawn by the authors.

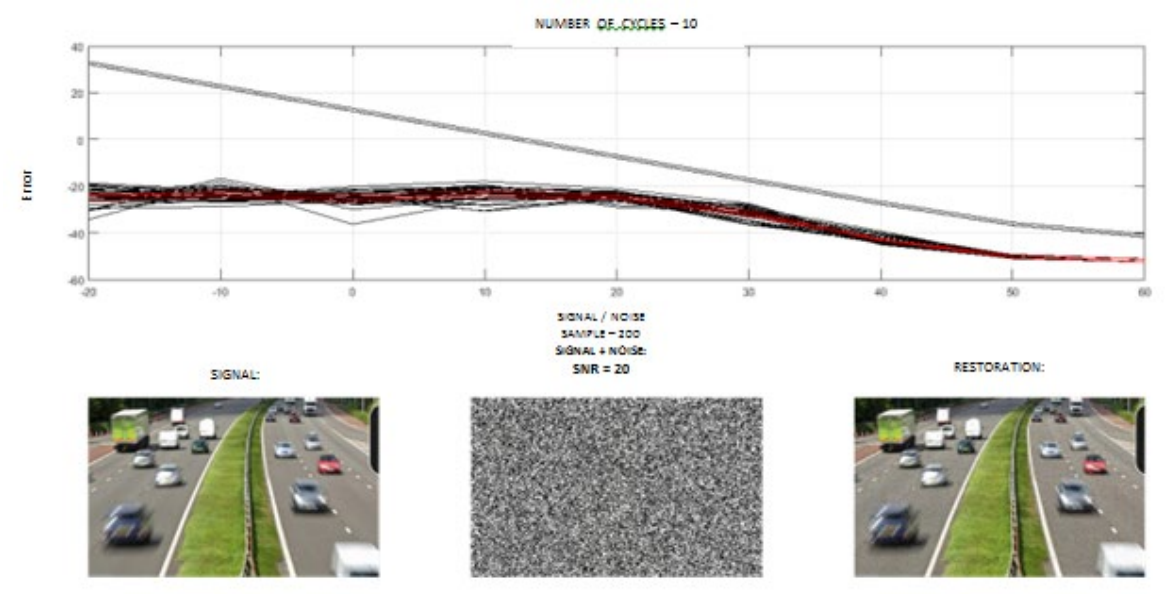

Fig. 2. Results of simulation of the functioning of the ICS RC in MATLAB environment Source: Drawn by the authors.

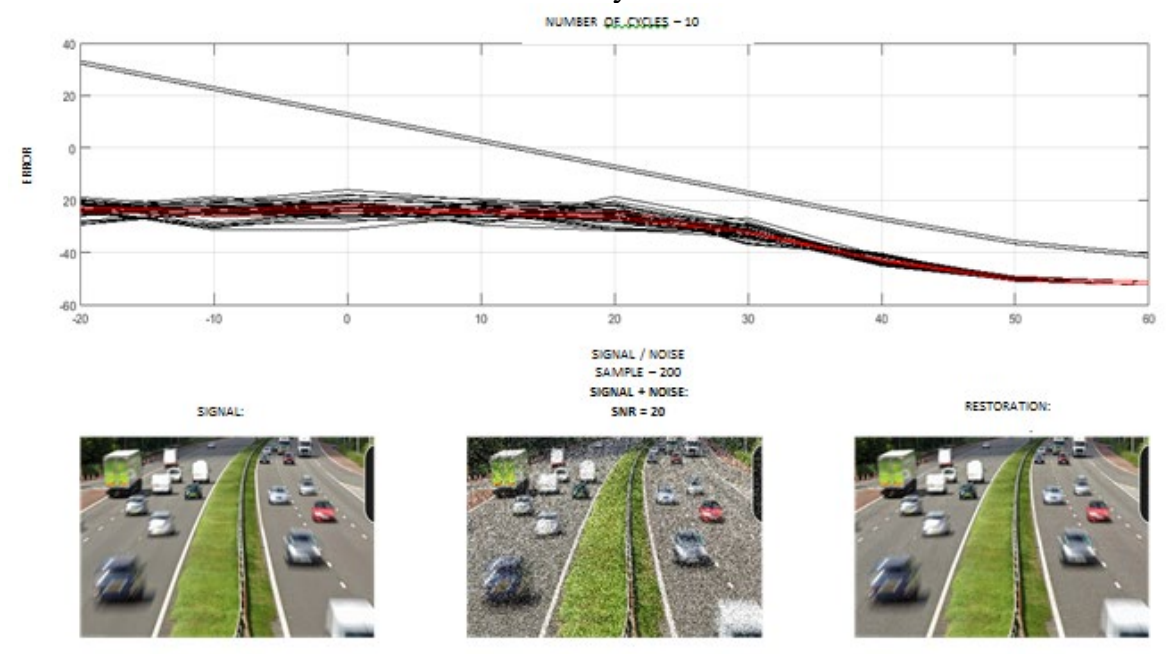

Fig. 3. Results of simulation of the functioning of the ICS RC in MATLAB environment Source: Drawn by the authors. 


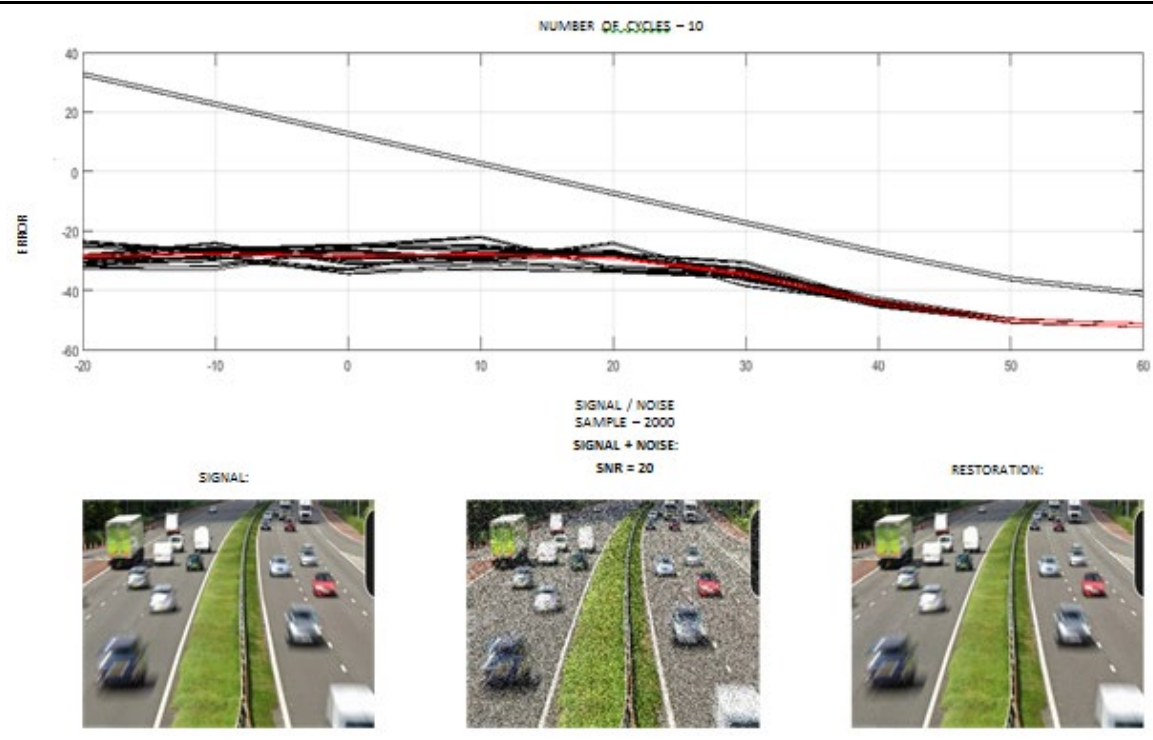

Fig. 4. Results of simulation of the functioning of the ICS RC in MATLAB environment Source: Drawn by the authors.

\section{CONCLUSION}

From the results of the analysis of the system of analytical expressions (14) - (16) there are several important directions concerning the specification of the domain of simulation of the process of adaptation of a robotic vehicle to destabilizing conditions of the operating environment. In particular, it is suggested to pay attention:

- on the determination of the criterion of optimal integration of information flows and the integration of multi-sensory channels of the ground robotic complex in the situation of redundancy of the information system,

- the admissibility of the application of the basic provisions of the theory of system adaptation in the class of optimization tasks related to minimizing the loss of information capabilities by ground robotic complex under the influence of external destabilizing factors of artificial origin,

- the necessity of searching in the class of optimal algorithms for adaptation of the ground robotic complex, least sensitive to destabilizing factors of intra-system origin, objectively present in any information system.

\section{REFERENCES}

[1] Skachkov, V., Bratchenko, G., Chepkiy, V., \& Yefimchikov, A. (2015). Entropy approach of information capabilities of a adaptive radio engineering system with intrasystem uncertainty. Electronics. 3-12.

[2] Klymenko, V., Polyakov, V., Sakno, O., \& Kolesnikova, T. (2018). Decision of informational problems in systems of technical vision of unmanned cars. Modern technologies in mechanical engineering and transport. 79. 
[3] KLIMENKO, V., \& SAKNO, O. (2018). Problem of informational interaction of unmanned car in uncertain and conflict conditions of exploitation. Kharkiv: Annual International Scientific and Practical Conference: New Technologies of Automobile Transport Development. 36-39.

[4] SkachKov, V., Bratchenko, G., Manko, V., \& Leshchenko, O. (2013). Sources of intrasystem distrurbances in information radio engineering systems. Scientific Production Journal. Kharkiv. Odesa. 222-225.

[5] Klimenko, V., Sakno, O., Moysya, D. \& Kotov, D. (2018). Problem of stability of solutions of information problems in systems of technical vision of vehicles. Collection of scientific papers of the Military Academy Odesa, 2018. 79 - 87. 